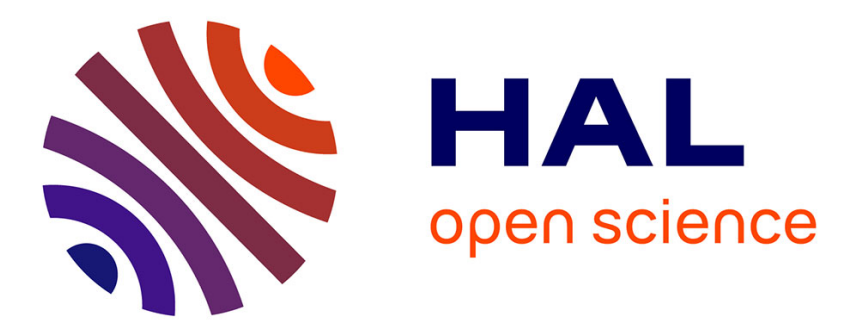

\title{
"L'Interdit de Valandraud ". La prescription et le droit dans le parcours d'un novateur du monde de production viti-vinicole bordelais
}

\author{
Pierre-Marie Chauvin
}

\section{- To cite this version:}

Pierre-Marie Chauvin. "L'Interdit de Valandraud ". La prescription et le droit dans le parcours d'un novateur du monde de production viti-vinicole bordelais. Terrains et Travaux: Revue de Sciences Sociales, 2004, nº 6 10.3917/tt.006.0013 . hal-03481181

\author{
HAL Id: hal-03481181 \\ https://hal.science/hal-03481181
}

Submitted on 15 Dec 2021

HAL is a multi-disciplinary open access archive for the deposit and dissemination of scientific research documents, whether they are published or not. The documents may come from teaching and research institutions in France or abroad, or from public or private research centers.
L'archive ouverte pluridisciplinaire HAL, est destinée au dépôt et à la diffusion de documents scientifiques de niveau recherche, publiés ou non, émanant des établissements d'enseignement et de recherche français ou étrangers, des laboratoires publics ou privés. 


\title{
«L'INTERDIT DE VALANDRAUD ». LA PRESCRIPTION ET LE DROIT DANS LE PARCOURS D'UN NOVATEUR DU MONDE DE PRODUCTION VITI- VINICOLE BORDELAIS (ENTRETIEN)
}

\author{
Pierre-Marie Chauvin
}

ENS Paris-Saclay | « Terrains \& travaux »

2004/1 nº 6 | pages 13 à 29

ISSN 1627-9506

DOI 10.3917/tt.006.0013

Article disponible en ligne à l'adresse :

https://www.cairn.info/revue-terrains-et-travaux-2004-1-page-13.htm

Distribution électronique Cairn.info pour ENS Paris-Saclay.

(C) ENS Paris-Saclay. Tous droits réservés pour tous pays.

La reproduction ou représentation de cet article, notamment par photocopie, n'est autorisée que dans les limites des conditions générales d'utilisation du site ou, le cas échéant, des conditions générales de la licence souscrite par votre établissement. Toute autre reproduction ou représentation, en tout ou partie, sous quelque forme et de quelque manière que ce soit, est interdite sauf accord préalable et écrit de l'éditeur, en dehors des cas prévus par la législation en vigueur en France. Il est précisé que son stockage dans une base de données est également interdit. 


\section{"L'Interdit de Valandraud ». La prescription et le droit dans le parcours d'un novateur du monde de production viti-vinicole bordelais}

(entretien)

"La vérité officielle que produit le travail collectif d'euphémisation, forme élémentaire du travail d'objectivation qui conduira à la définition juridique des pratiques convenables (...) a aussi une efficacité réelle, parce que, serait-elle démentie par toutes les pratiques, à la façon d'une règle de grammaire qui n'aurait que des exceptions, elle reste la vérité des pratiques qui se veulent convenables. "

Pierre Bourdieu, 1980. Le Sens pratique, Paris, Éditions de Minuit, p. 188.

Si le vin se veut un produit de qualité, il est tout d'abord un produit particulièrement "qualifié ». Un grand nombre d'acteurs (producteurs, négociants, critiques vinicoles, agents de l'INAO, etc.) sont amenés à le définir, le caractériser, l'évaluer selon des modes d'argumentations différents, qui ont une vocation, une portée, un statut variables. Parmi ces " qualifications » ${ }^{1}$, il en est certaines - les règles de droit - qui orientent d'une manière décisive les rapports possibles qu'entretiennent ces acteurs avec le produit. Ces règles sont en effet des qualifications relevant d'un ordre normatif singulier et servant de cadre d'action aux acteurs sociaux comme règles de conduite dont la sanction relève de la puissance publique. Dès lors

1 Ce terme est ici entendu dans son sens le plus large, c'est-àddire comme l'attribution d'une ou plusieurs caractéristiques au produit. Pour un examen plus approfondi de la notion de "qualification", voir notamment le récent dossier consacré à la qualité des produits, dans Sociologie de travail, 2002, 44, pp. 255-287. 
que l'on s'intéresse à l'activité économique (des professionnels du vin), elles apparaissent comme un élément central d'orientation des conduites. Dans un contexte de concurrence sur les marchés, elles fonctionnent non seulement comme des contraintes mais aussi comme des ressources, à ce titre visées par les acteurs. Les règles juridiques, appréhendées en leur sens sociologique, peuvent être considérées comme "des références pour les actions, qui sans prendre nécessairement celles-ci pour objets et à proprement parler, les "diriger", concourent par diverses voies, et avec une intensité variable, à leur gouvernement" (Jeammaud, 2000, pp. 224-225). Pour mettre en lumière les effets socio-économiques des règles de droit dans la sphère viti-vinicole, il faut d'abord les resituer dans la constellation des normes qui qualifient les produits et orientent les activités productives. C'est là une spécificité vinicole: le vin est non seulement un produit qualifié mais aussi et surtout un produit hiérarchisé. Il existe ainsi un certain nombre de classifications qualitatives qui établissent les rapports d'équivalence entre les vins. Il est donc essentiel de distinguer les différents types de normes et de hiérarchies qualifiant ces produits.

Notons d'abord l'existence de normes publiques, définissant un mode de valorisation légitimé par l'État. Le système viti-vinicole français est en grande partie caractérisé par la grille normative publique que constituent les Appellations d'Origine Contrôlées. En instituant la valeur des vins à partir de critères géographiques, productifs et gustatifs, les AOC ont une double vocation. Elles concernent le producteur dans la mesure où elles régulent la concurrence et le protègent des "mauvais" vins ${ }^{2}$ qui pourraient encombrer le marché ${ }^{3}$, et lui permettent de valoriser son produit. Le corollaire de cette valorisation instituée légalement est qu'elle est censée fournir un repère qualitatif au consommateur, dans une exigence de transparence qui pourrait caractériser à certains égards l'engagement politique des questions de consommation tel qu'il

2 C'est-àdire des vins n'entrant pas dans certaines normes de qualité ou de typicité définies pour la région de production. Le terroir est censé constituer l'élément déterminant de la qualité du produit. Le plus souvent, les «mauvais » vins sont avant tout des vins « artificiels » ne respectant pas le subtil équilibre entre facteurs naturels et facteurs humains dans l'élaboration du produit, tel que le reconnaît I'Institut National des Appellations d'Origine (INAO). Rappelons que I'INAO est un établissement public administratif issu du décret-loi du 30 juillet 1935, qui instituait initialement un Comité National des Appellations d'Origine, converti en juillet 1947 en Institut National.

3 Nous faisons ici référence aux analyses de G. Akerlof (1970) sur la possibilité que le «mauvais produit chasse le bon". 
apparaît au 20e siècle en France à partir de la loi sur la répression des fraudes de 1905 (Canu, Cochoy, 2003).

Par ailleurs, le vin est la source (et parfois le fruit) de qualifications privées émises par des critiques vinicoles décrivant et évaluant les qualités organoleptiques du vin et par extension les qualités des propriétés viticoles et du travail qui y est effectué.

L'objet de cet article est de montrer comment ces différents types de qualifications "orientent" les stratégies des producteurs, mais également comment ces qualifications se rapportent entre elles. À partir d'un entretien ${ }^{4}$, nous procéderons à la monographie d'un acteur $\mathrm{du}$ monde viti-vinicole bordelais, reconnu généralement comme un des acteurs à l'origine des "vins de garage ". ${ }^{5}$ Tout en ayant valorisé son vin en grande partie à partir des notations de prescripteurs privés (guides et critiques vinicoles), et étant l'un des seuls producteurs de vins du Bordelais à jouer le temps d'un millésime (et «l'espace» d'une partie de ses parcelles de vignes) la carte du déclassement (hors AOC), cet hérétique affiché reste fortement inscrit dans la hiérarchie historiquement instituée des classements officiels et des normes publiques des AOC dont il mobilise, parfois en creux, toutes les ressources.

\section{Un nouvel entrant dans le monde de production bordelais}

"En 1989, J.L.T. et sa femme M.A. acquièrent une petite parcelle de 0,6 hectare dans le vallon de St-Émilion entre Pavie-Macquin et La Clotte. Ils réalisent en 1991 leur premier millésime mis en bouteille par leurs soins. (...) Le château de Valandraud, bien que non classé, est considéré par presque tous les professionnels du vin, dont Robert

\footnotetext{
4 Réalisé dans le cadre d'un mémoire de maîtrise, Produits qualifiés, stratégies orientées. Le cas des vins du Bordelais, Université Paris X - ENS de Cachan, 2003.

5 Depuis la fin des années 1980, apparaît au sein du monde de production bordelais une nouvelle classe de produits, fondée sur quelques éléments caractéristiques : produits en très petite quantité (on les appelle aussi micro-cuvées). Objets d'une spéculation et d'une médiatisation spectaculaires, ces vins, présentés le plus souvent comme le résultat d'un travail d'orfèvre, peuvent être vendus à des prix plus élevés que les Premiers Grands Crus classés.
} 
Parker et René Gabriel, comme l'un des tous premiers crus de Bordeaux."

La trajectoire de JLT est marquée par une entrée récente dans le monde de production viti-vinicole bordelais et par le souci de faire rapidement partie des "référentiels " bordelais, ces Grands crus qui se vendent à prix d'or, et dont le prestige s'étend bien au-delà des sphères viticoles. L'entrée de ce producteur dans le segment de prix le plus élevé des vins de Bordeaux, s'il est un phénomène marginal au sein du monde de production bordelais, ne constitue pas pour autant un fait anodin pour le sociologue.

La brève présentation ci-dessus, issue du site "Thunevin.com", montre l'importance du critique vinicole américain $R$. Parker dans la valorisation du vin considéré. Ce prescripteur constitue l'une des principales instances socialisantes du marché des vins de Bordeaux, en ce sens qu'il contribue fortement à la formation de l'offre, à la fois dans sa matérialité, en orientant les techniques de production en fonction d'une certaine idée qualitative, et dans son "immatérialité", en concourant à la formation des prix. Ce processus de formation (ou de socialisation) de l'offre se caractérise notamment par l'émergence d'une nouvelle catégorie de produits, que Parker a contribué à faire reconnaître comme une classe d'excellence auprès d'une certaine clientèle. ${ }^{6} \mathrm{Du}$ côté de la sphère productive, tous les acteurs viti-vinicoles ne se rapportent pas de la même manière à cette référence prescriptive : certains préfèrent ne pas faire goûter leur vin à Parker et considèrent cette ressource de valorisation comme trop incertaine (et donc trop risquée). D'autres ont au contraire bâti leur réputation en grande partie sur les évaluations positives du critique américain. C'est d'ailleurs l'une des caractéristiques communes des nouveaux entrants du monde de production bordelais regroupés dans la catégorie des "vins de garage" que de s'appuyer sur les évaluations de prescripteurs pour valoriser commercialement leur vin, à défaut de pouvoir prendre appui sur le système de classement traditionnel. ${ }^{7}$

6 Nous retrouvons ici l'un des effets socio-économiques de l'activité prescriptive, analysé par A. Hatchuel (1995).

7 Cette hiérarchie traditionnelle est constituée de classements professionnels reconnus par l'État, le plus connu d'entre eux étant le classement de 1855 - non révisable, contrairement à d'autres classements comme celui de Saint-Émilion, révisable lui tous les dix ans -, qui établissait initialement une liste des vins les plus réputés (et les plus chers) de la Gironde, et qui valorisa, de fait, uniquement des crus du Médoc (à l'exception de l'un d'entre eux). 
La dénomination "vins de garage" est apparue dans les discours d'acteurs traditionnels du monde de production bordelais ainsi que dans les médias, en référence au garage de JLT, converti en chai de vinification faute de locaux spécifiques. La connotation péjorative et le mépris associé à l'impureté d'un tel espace productif ont rapidement été doublés d'une nouvelle interprétation du terme, plus positive, car résultant de son appropriation par les acteurs jusque-là stigmatisés. Voyons comment JLT se positionne lui-même à l'égard des autres producteurs :

"Certains s'appuient sur leur Histoire. C'est les B. et compagnie... La propriété se transmet depuis deux ou trois générations, et on hérite de ça, on en jouit. Moi, j'ai créé ma propre histoire. Bien sûr j'ai mon histoire, mon père a été résistant dans le maquis, je suis pied noir, j'étais employé de banque, mais cette histoire elle ne m'a pas servi pour faire mon vin. Moi, je leur demande à ceux qui héritent et qui font sans cesse référence à la fidélité à un terroir, à une tradition, à la famille, au père, au grand-père...ce qu'ils ont fait eux-mêmes. Mais attention! Le terroir n'exclut pas forcément le travail des hommes et réciproquement. Simplement, quand on a un grand terroir, c'est plus facile de se reposer sur ses lauriers que de les remettre en jeu. Quand j'ai commencé, on m'a accusé de ne pas avoir de terroir. On a confondu le fait que je n'étais pas un héritier et le fait que je n'avais pas de terroir. D'ailleurs, les parcelles de SaintÉmilion appartiennent à une aire de production, ce n'est quand même pas moi qui y suis pour quelque chose! Moi je n'ai pas de diplôme, pas d'intelligence pure, pas un QI formidable, juste du bon sens, et j'ai voulu faire ce que je pouvais faire dans un domaine: celui du vin."

La stratégie de présentation de soi s'appuie ici sur un principe d'opposition : JLT oppose les "héritiers", porteurs d'une histoire familiale agissant sur les hommes plus qu'elle n'est " agie" par eux, à sa propre trajectoire, qui est celle d'un homme dont la généalogie n'a pu nourrir les capitaux et les dispositions nécessaires à l'action viti-vinicole. Tout se passe comme si son histoire était scindée en deux, avec un passé a-vinicole qui ne lui aurait servi en rien dans son activité actuelle, et un destin vinicole qu'il serait en train de construire, indépendamment de ce qu'il a pu vivre auparavant. Pourtant cette histoire a-vinicole est remobilisée dans le cadre de sa "nouvelle» histoire: les éléments biographiques évoqués (père 
résistant, pied noir, employé de banque) fonctionnent comme des ressources de légitimation d'une trajectoire atypique, qui peut être valorisée comme telle au sein d'un monde de production réputé pour sa clôture et son conservatisme.

Le travail accompli est donc ce qui permet à notre interlocuteur de valoriser sa propriété et son vin, par opposition aux jouisseurs de grands terroirs, qui ont le privilège des réputations acquises et des reconnaissances officielles. Cependant, même si d'aucuns jugeront ses parcelles moins nobles que d'autres parcelles mieux exposées et/ou structurées, elles appartiennent à l'aire de production délimitée par l'INAO, ce qui lui permet de répondre aux critiques portant sur la médiocrité de son terroir. Lui aussi peut valoriser la qualité de son vin par son inscription géographique et géopédologique, notamment à partir du nom de Saint-Émilion. Ce que nous dit cet acteur, c'est que le Bordeaux viti-vinicole n'aime pas les succès rapides de vins non reconnus par les hiérarchies officielles. Du moins cette affirmation lui permet-elle de jouer la carte de « l'hérétique».

Cette façon de présenter sa trajectoire n'est pas simplement une posture, elle se traduit par des actions matérielles (techniques d'élaboration). Mais celles-ci sont toujours mises en scène et unifiées autour de la figure de l'innovateur, ce qui apparaît non seulement dans l'entretien réalisé, mais aussi au cours des différentes expositions médiatiques qu'ont connues JLT et sa femme. Nous allons à présent voir comment cette présentation de soi renvoie à des méthodes de production originales, pour évaluer enfin la manière dont l'activité de cet "hérétique" reste orientée par les normes, y compris les plus traditionnelles. 


\title{
Techniques de production et jeu avec les normes
}

\author{
Des techniques de production orientées vers une idée \\ qualitative : l'innovation et le spectre du fabricateur
}

JLT le répète inlassablement: il est un innovateur, ne cesse de (se) poser des questions, entreprend tous les essais qu'il peut, du moment que cela est au profit de la " qualité ".

\begin{abstract}
"Nous, de toute façon, on a tout essayé et on essaiera tout. (...) En général, ce ne sont pas des essais pour des essais, parce qu'il y a des gens qui font des essais pour faire des essais, des chercheurs, vous connaissez ça? Il y a des gens qui passent leur vie à faire des études, sans jamais aboutir, c'est comme un mec qui drague mais qui ne veut pas passer à l'acte. Ils sont des essayeurs. Nous on n'est pas des essayeurs, si j'essaie un truc c'est que j'espère trouver par là quelque chose de mieux."
\end{abstract}

La nature manuelle des pratiques de viticulture déployées est fondamentale, car elle imprime une identité artisanale voire artistique au produit. Elles peuvent donc être exposées publiquement et constituer un atout commercial à utiliser sur différents supports de valorisation. On trouve ainsi sur le site Internet de la propriété une présentation détaillée des techniques viticoles utilisées, rythmant le travail de la vigne. Les techniques présentées témoignent d'une volonté explicite de concentration des vins produits. Une grande partie du travail repose sur la façon dont on "fait souffrir" la vigne, par des opérations comme les vendanges vertes (réalisées courant juin). Celles-ci consistent à éliminer trois grappes sur quatre, afin de faire passer les rendements de 60 à 20 hectolitres par hectare. ${ }^{8}$ En concentrant l'énergie de la vigne sur quelques grappes on obtient des raisins riches, avec une taille de grains particulièrement faible, ce qui est un signe de qualité. De même on opère un effeuillage partiel du côté du soleil levant, et un effeuillage total trois semaines avant les vendanges, afin d'exposer les raisins aux rayons du soleil, garants d'une maturité supérieure. Dans ces conditions de production, les quantités produites sont bien évidemment très faibles, ce qui confère au produit une rareté

8 Le rendement est ainsi devenu un référent qualitatif presque incontournable, comme si un faible rendement était le seul garant de la qualité du produit, contrairement à ce que s'attachent à montrer des Grands Crus Classés aux rendements plus élevés. 
entretenant les phénomènes spéculatifs. Mais si cette rareté permet de donner au produit le statut de produit artistique ou de luxe, elle n'est pas l'objectif ultime du producteur : celui-ci aspire au contraire à produire plus, mais toujours au même prix et avec la même qualité. Le défi n'est pas de rejoindre ces Grands Crus du Bordelais qui peuvent vendre dans le même segment de prix des vins produits dans des quantités jusqu'à cent fois supérieures, mais il s'agit de s'en approcher, dans les limites imposées par la taille de l'exploitation. Ainsi JLT explique ses ambitions quantitatives :

"Aujourd'hui, comme j'aspire à un statut plus historique, j'essaie que mes quantités ne soient pas considérées comme trop minimes; d'ailleurs à 15000 bouteilles par millésime, ça tient la route. Il faudrait que j'arrive à faire des volumes comme Ausone, pour voir si mon produit est toujours au même prix avec ce genre de volume."

La référence au château Ausone, Premier Grand Cru Classé de Saint-Émilion, est un premier signe des limites de la posture de chef de file des vins de garage. ${ }^{9} \mathrm{La}$ formule clé est bel et bien: "Aujourd'hui, j'aspire à un statut plus historique. " En effet, la carte de l'innovation, lorsqu'elle est instrumentalisée de façon systématique dans les actions de valorisation, comporte certains risques. Ces risques sont ceux d'une déviance relative à certaines normes sociales ou juridiques. Ces deux types de normes sont particulièrement présents dans le monde viti-vinicole bordelais, et l'innovateur est conduit à les ressentir à travers les sanctions du groupe porteur de la norme considérée. En ce qui concerne la norme "sociale", le groupe porteur est constitué des acteurs traditionnels $\mathrm{du}$ monde de production bordelais. Pour prendre un exemple de l'influence de ce type de norme sur un acteur viti-vinicole, citons ces quelques mots de JLT, qui en disent long sur les critiques rencontrées au cours de sa carrière viticole:

"On était suffisamment originaux avec Valandraud, suffisamment empêcheurs de tourner en rond, il faut pas en rajouter, suffisamment typés "pas de terroir", suffisamment typés "fabricants de vins" pour ne pas augmenter ça."

En somme, la norme sociale mobilisée ici, fondée sur le continuum

9 Cette référence n'est pas anodine, A.V., gérant du Château Ausone, étant associé àJLT, tous deux ont par conséquent des intérêts économiques communs. 
qui va du terroir à la fabrication, n'est pleinement manifeste et efficace que dans les situations de litige, où elle prend appui sur la norme juridique constituée par l'AOC. JLT se présente volontiers luimême comme trop "fabricateur ", sa crainte n'est formulée qu'après l'évocation d'une forme de litige avec l'INAO. Ne pas "augmenter" son image de fabricateur signifie ne pas aller trop loin dans la transgression de certaines normes juridiques, dont le groupe porteur est l'INAO. Une innovation technique l'a en effet conduit à être sanctionné par cet organisme administratif, à travers le déclassement d'une partie de ses parcelles en vin de table. La norme sociale de fabricateur peut dépendre d'une norme publique, exprimée à travers cette sanction étatique qu'est le déclassement. Mais le producteur peut jouer avec ces normes juridiques dont l'effectivité sociale ne se manifeste pas seulement sous le mode de la sanction ou de la contrainte. Il est possible de se situer à leur marge, et de se servir de cette distance comme d'une ressource d'action.

\section{L’ambiguïté du déclassement : entre sanction publique et ressource marketing}

- Peut-on revenir sur le déclassement, vous avez bâché vos vignes?

- C'est une anecdote. On a fait ça deux ans, et on a continué à le faire. On avait le droit de faire des essais en 1998, en 1999, et en 2000 ils n'ont plus voulu.

- C'est l'origine du déclassement?

- D'une parcelle. Je regardais l'autre jour sur un site Internet, il y avait marqué "il s'est brûlé les ailes avec le déclassement". Bon, ce n'est pas vrai parce qu'en fait on a vendu, je ne dis pas que c'est bien, mais on a vendu quand même des bouteilles, c'était un autre produit qui était sur le marché, et on a gagné plus d'argent - ça je ne peux pas le dire, mais bon...

Ces essais, pourquoi on fait ces essais? On savait qu'il y avait des essais qui se faisaient à Margaux, à Cheval-Blanc et à Petrus... Donc on s'est demandé pourquoi eux font des essais ? On a cherché, on n'a pas trouvé la première année, mais la deuxième année on a trouvé. J'étais emballé par le système, en bâchant les vignes on empêchait l'eau de rentrer, c'est pas une invention extraordinaire. La plupart des fraisiers sont comme ça...

- Il y a une réglementation qui l'interdisait?

- Alors on a eu l'autorisation de faire des essais, c'est une 
autorisation non demandée, comme la plupart des autres en plus... À Bordeaux, c'est comme ça il vaut mieux faire en douce. On a eu des réunions avec le syndicat, l'INAO et la Chambre d'Agriculture, on nous a dit " si le vin est mauvais, il est mauvais, c'est le climat, le terroir qui le veut». (...) Le déclassement pour moi n'était pas une punition, on m'a dit « il ne faut pas faire ces essais », j'ai dit « je veux continuer à faire des essais". Première année autorisée, deuxième année autorisée, troisième année pas autorisée...

- Pourquoi?

- Pourquoi ? Pas d'explication. Le déclassement avait été averti, c'est moi qui ai pris la décision d'être déclassé, mais pourquoi on n'avait pas le droit de faire ces essais... Il y a eu suffisamment d'essais.

- Comment vous est venue l'idée d'appeler votre vin "l'Interdit de Valandraud"? Ce nom a fait parler de votre vin, c'est un nom qui sort du lot?

- Le seul problème de l'Interdit de Valandraud, là pour le millésime 2000 , c'est que le vin est moins bon que le Valandraud. Si j'avais fait meilleur, j'aurais pu jouer un peu le pauvre que l'on égorge, que l'on soumet à des règles drastiques, ce pauvre petit chaperon rouge qui est perdu dans une forêt... Je ne pouvais pas, parce qu'en fait, moi j'avais fait mes essais, ces essais je les ai faits normalement, la parcelle où on a fait ces essais n'a pas bien fonctionné cette annéelà, et on a fait un vin bon mais moins bon. On l'a vendu au même prix. La spéculation, c'est pas toi qui la décide. Le fait de les avoir vendus cher n'est déjà pas mal, le fait que les négociants bordelais qui en ont acheté et qui les ont revendus, c'est bien... Aujourd'hui les bouteilles de Valandraud on aura du mal à les trouver, sur 15 000, c'est pas beaucoup, mais c'est déjà pas mal... mais je suis loin du score de " Le Pin ", là il faut batailler...

Notre interlocuteur commence par minimiser l'affaire, en la renvoyant au statut de l'anecdote. C'est un premier indicateur du risque qu'il y a pour un acteur du monde de production bordelais à être impliqué dans un litige juridique, quelle qu'en soit la nature. L'intensité des relations sociales et professionnelles de ce district bordelais, ainsi que l'exposition médiatique de cet espace productif concourent à des phénomènes de circulation de l'information particulièrement rapides et dangereux pour qui en fait l'objet. Ainsi l'image de "fabricateur" de vins et l'idée de "vins de garage" se sont propagées de manière assez efficace pour que le producteur stigmatisé veuille en freiner la diffusion. 
À côté de cette minimisation, l'acteur cherche à valoriser ce qui peut l'être dans le déclassement, c'est-à-dire la part d'autonomie inhérente à l'acte de transgression. Il déplore le fait que des journalistes en soient restés à la surface des choses, c'est-à-dire à la dimension coercitive liée à la sanction du déclassement. Il est vrai que ce type d'affirmation péremptoire ("il s'est brûlé les ailes ») n'est pas rare, et laisse surtout dans l'ombre la façon dont un acteur économique peut jouer avec les normes juridiques. Derrière l'hypostase de la sanction, qui tombe comme un couperet sur la tête du déviant, il y a la vision commune de la règle juridique comme contrainte. Or ce cas montre précisément qu'il y a une autre façon de concevoir le rapport aux normes. Qu'il faille parler de stratégie $a$ priori ou de conversion a posteriori d'une contrainte en ressource, il est clair que ce producteur ne peut être considéré comme un simple agent subissant une intervention étatique. Certes il lui arrive de mettre en scène la sanction du déclassement comme une décision arbitraire ("En 2000 ils n'ont plus voulu. Pourquoi? Pas d'explication. "), mais il entend avant tout montrer qu'il est resté maître de la situation, que ce déclassement est le fruit de sa volonté, voire d'un choix rationnel ("C'est moi qui ai pris la décision d'être déclassé"). La question qui se pose est alors: pourquoi avoir " accepté » ce déclassement? Différents éléments de réponse peuvent être mobilisés.

Ce déclassement était certes acceptable car il ne concernait qu'une partie des parcelles de l'exploitation. Le risque encouru n'était donc pas majeur, car une grande partie de la production pouvait continuer à être vendue en AOC Saint-Émilion, sous la dénomination habituelle de Château Valandraud. Si les bouteilles déclassées en vins de table n'avaient pu être commercialisées au même prix, ou même ne pas trouver d'acheteurs, l'autre partie aurait permis d'assurer un chiffre d'affaires satisfaisant. Mais le scénario a été bien différent, car les bouteilles déclassées se sont vendues... au même prix que celles valorisées par l'AOC ! Comment cela a-t-il été possible, alors que de l'aveu de JLT lui-même la qualité du vin déclassé était inférieure à celle du vin commercialisé en AOC ? Cette réussite commerciale revient dans une certaine mesure à l'habileté marketing du viticulteur, aidée par la créativité d'un de ses fidèles clients (l'un des dirigeants de Farr, grande maison de négoce londonienne). En effet, celui-ci lui conseilla d'appeler son vin 
"L'Interdit de Valandraud», en jouant ainsi avec la sanction étatique, et en la convertissant en ressource de valorisation. On est loin du coup de guillotine évoqué par certains. En quelque sorte, JLT a bien usé, contrairement à ce qu'il affirme, de la stratégie " $d u$ pauvre que l'on égorge, que l'on soumet à des règles drastiques..." Cette stratégie pourrait entrer en tension avec la mise en valeur de son autonomie transgressive. En fait le jeu avec la norme publique est ici le même : d'un côté jouer les victimes en soulignant l'arbitraire de la décision étatique et en affichant publiquement la sanction subie ("L'Interdit...») ; de l'autre éclairer sa propre responsabilité afin de convertir la victime en déviant conscient et maître de sa transgression, pour faire un élément valorisant au niveau commercial.

\section{Déclassé, socialisé : l'orientation de l'activité économique par les normes publiques}

En première analyse, on pourrait dire que le temps d'un millésime et l'espace d'une parcelle, JLT a joué la carte du marché plutôt que celle de la norme. Il a choisi de sortir une partie de sa production du système de valorisation institué par l'État que constitue l'AOC, et de laisser le marché "valoriser" son vin. La dénomination "vin de table" n'étant pas vraiment un signe de qualité auprès du consommateur, cette valorisation passe en grande partie par l'appui sur des prescripteurs privés. Cet appui est notamment visible sur le site de JLT, qui offre un tableau récapitulatif des différentes évaluations des critiques vinicoles pour chaque millésime du Château Valandraud. Ces notes des critiques vinicoles constituent un certain type de prescription, sachant qu'il y a d'autres ressources de valorisation: nous avons évoqué l'innovation marketing liée au nom, mais il faudrait évoquer également le rôle des prescripteurs traditionnels que sont les négociants bordelais. JLT signale d'ailleurs l'importance de tels acteurs, qui, en déposant leur "griffe" sur le produit, renforcent son identité bordelaise et peuvent accroître sa légitimité socio-économique, si la maison de négoce a une solide réputation. Il est lui-même négociant, mais a besoin des réseaux de commercialisation bordelais pour vendre son vin, principalement 
acheté par de riches clientèles étrangères (particuliers et restauration). Ainsi il parle d'une maison de négoce bordelaise spécialisée dans les Grands Crus :

"Si on prend la maison X. Cette année, elle ne m'a pas acheté de Virginie [second vin du Château Valandraud], ou pas beaucoup. En volume, cette année on a dîu faire 12000 bouteilles, ou 15000 ou 20 000, donc si elle ne nous en a pas acheté ce n'est pas parce qu'il y avait trop de vins, c'est uniquement parce que le prix était trop élevé. Qu'est-ce que c'est un prix trop élevé pour cette maison? C'est quand on n'arrive pas à le revendre. Point. Et quand on me dit, "je ne peux pas les acheter", je dis c'est normal. En plus la maison X, je lui dois beaucoup, c'est plus important que Parker... Eh oui! La crédibilité d'un produit se fait par les grands professionnels, et la maison $X$, c'est un grand professionnel!"

Les "grands professionnels", qu'ils soient négociants ou critiques vinicoles (ou encore courtiers, journalistes, cavistes...) font la crédibilité et la croyance dans les produits (Bourdieu, Delsaut, 1975). L'enjeu pour le producteur est d'être reconnu par le plus grand nombre de ces professionnels du marché. Cependant, cet appui prescriptif n'est pas suffisant, en ce sens que la valorisation par les $\mathrm{AOC}$ et les classements afférents sont jusqu'à présent indispensables (au moins dans le Bordelais). On le voit bien avec ce producteur qui n'entend absolument pas poursuivre sa trajectoire viti-vinicole d'hérétique, aspirant plutôt à être mieux reconnu par le classement officiel de Saint-Émilion et faire partie des « référentiels » bordelais :

"Soit c'est une fumisterie et ça ne peut pas durer, soit c'est sérieux et ça doit durer ; alors, comment on peut être meilleur que les autres? Comment se fait-il qu'au bout de 10 ans on soit encore aussi bon, mieux que Lafite, Mouton...? (...) Moi je m'adapte un petit peu aux règles, je ne veux pas faire comme ces gars qui disent "je veux être Premier Cru”, sans passer par les Grands Crus Classés, je préfère être Grand Cru Classé avant d'être Premier Cru, il faut attendre 2005. Que l'on m'accepte ou que l'on ne m'accepte pas, dans tous les cas je serai gagnant. Si on m'accepte j'augmente ma réputation, si on ne m'accepte pas, tout le monde va dire "c'est pas normal", il y aura un vrai débat, qui va les embarrasser quand même..." 
L'ambition de JLT est de monter progressivement les différentes étapes au sein de la hiérarchie traditionnelle des vins de Bordeaux. ${ }^{10}$ Cette forme de légalisme manifeste l'intériorisation de schèmes cognitifs "orientés" par le droit. En ce sens, on peut parler de socialisation de la stratégie productive par une partie du droit vitivinicole, c'est-à-dire par des dispositifs juridiques dont le garant est la puissance publique. Au final, la socialisation de l'activité économique par les prescripteurs privés ne se substitue pas à l'instance socialisante constituée par les AOC comme figure juridique de l'État. Parker n'aurait pu gagner l'importance sociale qui lui est reconnue sans s'appuyer sur ces repères essentiels que sont les Appellations d'Origine Contrôlée et les hiérarchies qui en dépendent. On aime à dire en Bordelais que Parker a beaucoup fait pour Bordeaux (en termes de valorisation), mais la réciproque est aussi vraie : Bordeaux a beaucoup "fait" pour le succès de Parker. En effet, la structure juridique de l'univers viticole bordelais, et les repères cognitifs qu'elles fournit, ont été des points d'appui essentiels aux "prises" (au sens de Bessy, Chateauraynaud, 1995) réalisées par Parker, qui, entre conformisme et distinction, a su se positionner par rapport aux conventions qualitatives existantes (et jouer avec elles). Ces repères cognitifs sont précisément davantage que des repères de connaissance : ils agissent, ou plutôt insufflent à l'action humaine un air particulier. Le producteur vinicole "déclassé » n'en reste pas moins socialisé par la norme dont il s'est affranchi. Pour comprendre cette socialisation, référons-nous aux analyses de Max Weber à propos du « tricheur " et des " partenaires économiques » :

"Une "orientation" de l'activité d'après un règlement établi peut aussi consister en ce qu'un des individus socialisés agit sciemment à l'encontre du sens du règlement, tel qu'il est compris subjectivement; ainsi celui qui "triche" reste néanmoins socialisé comme "partenaire", contrairement à celui qui se retire du jeu. Il en est exactement de même du "voleur" et de l' "assassin" qui, tout en violant de façon consciente et subjectivement significative les règlements, orientent néanmoins leur comportement d'après ces règlements, par le fait même qu'ils dissimulent leurs actes ou leur personne." (Weber, 1992, p. 324).

Le producteur déclassé a-t-il triché avec les règles collectives, a-t-il dissimulé ses actes ? Et est-il donc toujours socialisé ? Au premier

10 Ce qui n'est d'ailleurs possible que du fait du caractère révisable du classement de Saint-Émilion. 
regard, il pourrait sembler que notre cas ne corresponde pas au mécanisme évoqué par Weber, le producteur n'étant pas un tricheur ayant dissimulé ses actes, mais un acteur qui choisit délibérément de sortir d'une norme (c'est-à-dire du jeu). L'acteur en question reste pourtant socialisé par l'AOC: une partie de sa récolte est toujours commercialisée en AOC Saint-Émilion et ce déclassement n'est que de court terme. Le fait de se retirer partiellement du jeu n'implique pas une "désocialisation" du partenaire économique, mais témoigne d'un jeu avec la norme, dont les limites sont testées, éprouvées, afin de la convertir en ressource productive. Ainsi le déclassement est converti en ressource de valorisation par le producteur dans la mesure où il est marginal, temporaire, et que lui succède un retour au légalisme qui va structurer profondément la stratégie future de l'acteur.

La socialisation de l'action économique par le droit est donc nécessairement polymorphe. Ses différentes formes sont autant des orientations " à partir " de la norme que des jeux " avec" la norme. Le lien créé, de prime abord, concerne deux acteurs : l'État et l'acteur économique. Mais ce lien est aussi un lien public entre les partenaires économiques que sont les producteurs vinicoles (et indirectement les courtiers et les négociants), car il crée un cadre commun d'orientation des activités individuelles. Même si celles-ci diffèrent, même si les "jeux" avec la norme sont inégalement distribués socialement (le pouvoir coercitif de la norme est pour certains beaucoup plus fort que pour d'autres), il demeure cette conclusion essentielle, qui va à l'encontre des hypothèses classiques d'une sociologie de l'action oublieuse de l'intuition commune aux "pères fondateurs" de la sociologie que sont Durkheim et Weber ${ }^{11}$ : les dispositifs juridiques orientent (socialisent) les activités économiques plus qu'ils ne les contraignent. Et pour plagier une formule wébérienne sur l'importance de la problématique des biens (ou des produits) dans toute analyse sociologique des activités économiques ${ }^{12}$, on pourrait affirmer qu'une théorie sociologique du produit ne peut se dispenser d'inclure dès ses premiers pas la notion de "droit" ou de dispositifs juridiques qualifiant le produit, ces

11 Bien que cette commune intuition soit développée de manière bien différente par les deux sociologues. 12 Rappelons cette phrase extraite d'Économie et société : "Une théorie sociologique de l'économie ne pourra se dispenser d'inclure dès ses premiers pas la notion de "biens" dans ses catégories (...), car elle a pour objet une "activité" qui reçoit sa signification spécifique du résultat (...) des réflexions des agents économiques". (1995, tome 1, p. 108). 
qualifications étant le principal facteur d'orientation des activités liées à son élaboration et à sa valorisation.

L'idée d'orientation des activités par les règles de droit nous paraît être une intuition particulièrement féconde pour qui veut saisir conjointement les ressorts sociaux des produits, des actions économiques présidant à leur élaboration, et des institutions socialisantes dans lesquelles elles s'inscrivent (et dont elles peuvent vouloir s'affranchir). Cette problématique, en prenant en considération à la fois le sens visé par les acteurs dans l'univers des règles de droit et le poids structurant de celles-ci sur leurs stratégies, permet d'échapper à l'alternative qui semble enfermer toute sociologie de l'action: à savoir l'individualisme méthodologique versus le holisme.

En étudiant comment les règles orientent les actions de professionnels d'un produit du terroir, on aura éclairé ce qui peut échapper à l'amateur, au professionnel, ou même à l'analyste plongé dans des considérations uniquement techniques ou symboliques : les règles sont des ressources qui, elles aussi, produisent le vin.

Pierre-Marie Chauvin pmchauvin@aol.com

\section{RÉFÉRENCES}

AKERLOF (G. A.), 1970. "The market for Lemons: qualitative uncertainty and the market mechanism ", Quarterly Journal of Economics, 84, pp. 488-500.

BESSY (C.), CHATEAURAYNAUD (F.), 1995. Experts et faussaires. Pour une sociologie de la perception, Paris, Métailié.

BOURDIEU (P.), 1980. Le Sens pratique, Paris, Éditions de Minuit. BOURDIEU (P.), DELSAUT (Y.), 1975. "Le couturier et sa griffe : contribution à une théorie de la magie ", Actes de la recherche en sciences sociales, 01, pp. 7-36.

CANU (R.), COCHOY (F.), 2003. «La loi de 1905 sur la répression 
des fraudes : un levier décisif pour l'engagement politique des questions de consommation?", Communication au séminaire de Sociologie Économique, ENS de Cachan.

HATCHUEL (A.), 1995. "Les marchés à prescripteurs. Crises de l'échange et genèse sociale ", in JACOB (A.), VERIN (H.) (dir.), L'Inscription sociale du marché, Paris, L'Harmattan, pp. 205-225.

JEAMMAUD (A.), 2000. "L'interdisciplinarité, épreuve et stimulant pour une théorie des règles juridiques", in KIRAT (T.), SERVERIN (É.), Le Droit dans l'action économique, Paris, CNRS.

WEBER (M.), 1992. Essais sur la théorie de la science, Paris, Plon/Pocket.

WEBER (M.), 1995. Économie et société, tome 1, Paris, Plon/Pocket. 